\title{
EDITORIAL
}

\section{Critical care resource allocation: trying to PREEDICCT outcomes without a crystal ball}

\author{
Michael D Christian*1, Robert Fowler², Matthew P Muller ${ }^{3}$, Charles Gomersall ${ }^{4}$, Charles L Sprung ${ }^{5}$, Nathaniel Hupert ${ }^{6}$, \\ David Fisman?, Andrew Tillyard ${ }^{8}$, David Zygun ${ }^{9}$ and John C Marsha110, on behalf of PREEDICCT Study Group
}

Despite pandemic influenza's long reign atop the list of potential medical catastrophes, the first protocol designed to support critical care triage in a pandemic was published only in 2006 [1]. Additional protocols followed, in attempts to address the goal of developing standardized, transparent and equitable tools for allocating critical care resources to those patients most likely to benefit [2-7]. Most of these protocols used the Sequential Organ Failure Assessment score as the quantitative underpinning for triage decision-making due to its ease of use. These protocols have been shown to generally direct resources to those most likely to benefit [8], in addition to making resources available for surge patients [9]. However, the Sequential Organ Failure Assessment score does not always differentiate well between survivors and nonsurvivors of critical illness for some patient populations $[10,11]$.

The International Forum of Acute Care Trialists (InFACT) was formed in 2009 and provided a platform for international critical care research collaboration during the 2009/10 influenza $\mathrm{A}(\mathrm{H} 1 \mathrm{~N} 1)$ pandemic [12]. Over the past 2 years, a number of working groups have emerged from InFACT focused upon improving the investigation and care of patients with severe respiratory illness. Arising from these efforts, in June 2012 an international group of clinicians convened the first meeting of the Providing Resources for Effective and Ethical Decisions In Critical Care Triage (PREEDICCT) Study Group. The study group's aim is to develop decision support tools appropriate for triaging critically ill adult patients during epidemics, mass-casualty scenarios or other resource-limited settings. This meeting identified a number of knowledge gaps and research priorities in this area, and suggested a revised framework for the requirements of an adequate triage decision support tool.

${ }^{*}$ Correspondence: Michael.Christian@utoronto.ca

'Department of Critical Care, Mount Sinai Hospital Toronto, 600 University Ave, rm 18-232-1, Toronto, ON, Canada, M5G 1X5

Full list of author information is available at the end of the article
While purpose-built triage protocols focus on specific events (for example, pandemics), resource allocation decisions are part of everyday practice for critical care physicians worldwide. Several PREEDICCT members work in settings where there are chronically insufficient critical care resources to meet the demand [13]. Critical care physicians also make resource allocation decisions every day in high-income countries, as they decide who might benefit from ICU care, when to accept outside transfers and when insufficient capacity dictates external transfer of patients. Yet intensivists lack objective tools to support these decision-making processes. Further, practices and specific decisions are likely to vary widely by country, by hospital and by individual provider.

The first significant shift in direction advocated by our group is to move away from attempting to use a physiologic score alone to predict outcomes. The rationale for basing triage tools on a physiologic score is that all critically ill patients compete for a single pool of critical care resources, regardless of whether they are part of the mass-casualty event or not [1]. However, there are at least two ways to compare different types of patients. The first method is to use the same tool to measure all patients, such as with a physiological prediction score (for example, the Sequential Organ Failure Assessment, Multiple Organ Dysfunction Score or Acute Physiology and Chronic Health Evaluation scoring systems). The second approach is to use different scores tailored to different diseases (for example, a burn score for a burn patient) that all produce a standard measure which can be compared. The potential benefit of using disease-specific scores, where available, is improved prognostication to overcome the deficiencies identified with generalized physiological scores.

For different predictive scores to be used when making resource allocation decisions, the scoring tool must allow comparison across different groups of patients based on a common metric. The PREEDICCT study group recommends that any such metric receive input from three important outcome dimensions: survival, quality of life, and resource consumption. Additionally, it is important to recognize that it is not an absolute measure of these 
factors which is primarily important but rather the incremental difference in the measure made by the provision of critical care resources [14,15].

A critical care resource allocation decision support tool based upon a combination of disease-specific and general physiological measures with common outcomes should be deployable in a variety of environments, including resource-limited countries. This utility probably requires a technological solution that combines often-complex scores into a single tool that facilitates rapid decisionmaking. The interface to facilitate this decision-making would use standardized categories of critical illness phenotypes (such as penetrating trauma, blunt trauma and pneumonia). Given the proliferation of mobile computing devices and the increasing presence of Internet access, we believe it will be feasible to create platform-independent software solutions that can meaningfully augment clinicians' capability to calculate and compare multiple variables in order to optimize utilization of potentially scarce critical care resources. Such tools will not supplant clinical decision-making; instead, they will provide additional data that clinicians can integrate with clinical experience to ensure that critical care admission is based on appropriate, clinically significant factors.

The first step in advancing this project will be to utilize the existing InFACT network in a truly global effort, involving both resource-rich and resource-poor countries, to better define current triage practices in high-income, middle-income, and low-income countries. Factors of interest include the type and frequency of resources and the method with which resource allocation decisions are currently made. Second, PREEDICT will survey existing disease-specific predictive scores - many of which presently only report a single endpoint, such as survival to determine whether crosscutting surrogate markers may permit alignment and comparison across disease categories. Once candidate metrics are identified and assembled into the tool, the team will use predictive modeling methodologies to forecast the impact of different thresholds for critical care admission on patient outcomes and also on facility-level and regional capacity and functioning. To be maximally useful, these modeling efforts will require richly descriptive data regarding health system functioning and patient outcomes, the types of which are increasingly being captured in stateof-the-art electronic medical records as well as research databases and registries.

To provide best care to patients during pandemics, environmental disasters or, indeed, day-to-day operations in resource-challenged settings, the global community of acute and critical care clinicians must increasingly see the practice of critical care as caring for critically ill patients, not only caring for patients in an ICU. When there are imbalances in demand and capacity, we should look to each other to help with context-appropriate ways to right this balance. When current and future challenges dictate that patient triage must occur, we must strive to develop decision-making tools to provide the optimal balance of survival and quality of life with the resources available.

\section{Abbreviations}

InFACT, International Forum of Acute Care Trialists; PREEDICT, Providing Resources for Effective and Ethical Decisions In Critical Care Triage.

\section{Competing interests}

DF has received grant funds and unrestricted educational funds from Novartis, GlaxoSmithKline and Sanofi-Pasteur vaccine divisions, all of which manufacture influenza vaccines. The other authors declare that they have no competing interests.

\section{Acknowledgements}

The views presented in this article are those of the first author (MDC) and do not represent the views of the Canadian Forces or Government of Canada. The PREEDICCT study group meeting was funded by a grant from the Canadian Institute of Health Research.

\section{Author details}

Participants of the PREEDICT Study Group Meeting (alphabetical order): Michael Christian, Canada; David Fisman, Canada; Robert Fowler, Canada; Charles Gomersall, Hong Kong; Margaret Herridge, Canada; Nathaniel Hupert, USA; Edgar Jimenez, USA; Peter Kraus, Canada; Bernard Lawless, Canada; Neil Lazar, Canada; John Marshall, Canada; Rudo Mathivha, South Africa; Seyed Moghadas, Canada; Mathew Muller, Canada; Lucy Pearmain, UK; Lewis Rubinson, USA; Charles Sprung, Israel; Henry (Tom) Stelfox, Canada; Andrew Tillyard, UK; Randy Wax, Canada; David Zygun, Canada.

\section{Author details}

'Department of Critical Care, Mount Sinai Hospital Toronto, 600 University Avenue, Room 18-232-1, Toronto, Ontario, Canada, M5G 1X5. ${ }^{2}$ Sunnybrook Health Sciences Centre, 2075 Bayview Avenue, Room D4 78, Toronto, Ontario, Canada, M4N 3M5. ${ }^{3}$ Infection Prevention and Control, St Michael's Hospital, 30 Bond Street, Toronto, Ontario, Canada, M5B 1W8. ${ }^{4}$ Department of Anaesthesia and Intensive Care, Prince of Wales Hospital, 30-32 Ngan Shing Street, Shatin, NT Hong Kong SAR. ${ }^{5}$ General Intensive Care Unit, Department of Anesthesiology and Critical Care Medicine, Hadassah Hebrew University Medical Center, PO Box 12000, Jerusalem, Israel 91120. 'Weill Cornell Medical College, 1300 York Avenue, New York, NY 10065, USA. 7 Hospital for Sick Children Research Institute, 123 Edward St, Room 428, Toronto, Ontario, Canada, M5G 1E6. ${ }^{8}$ Peninsula Medical School, Consultant Critical Care Medicine, Royal Cornwall Hospital, Treliske, Truro, Cornwall, TR1 3LJ, UK. ${ }^{9}$ Critical Care Medicine, 3C1.12 Walter Mackenzie Centre, 8440 - 112 Street, Edmonton, Alberta, Canada T6G 2B7. ${ }^{10}$ St Michael's Hospital, 30 Bond Street, Bond 4-014, Toronto, Ontario, Canada, M5B 1W8.

Published: 23 January 2013

\section{References}

1. Christian MD, Hawryluck L, Wax RS, CookT, Lazar NM, Herridge MS, Muller MP, Gowans DR, Fortier W, Burkle FM: Development of a triage protocol for critical care during an influenza pandemic. CMAJ 2006, 175:1377-1381.

2. Ashton-Cleary D, Tillyard A, Freeman N: Intensive care admission triage during a pandemic: a survey of the acceptability of triage tools. J Intensive Care Soc 2011, 12:180-186.

3. Christian MD, Joynt GM, Hick JL, Colvin J, Danis M, Sprung CL; European Society of Intensive Care Medicine's Task Force: Chapter 7. Critical care triage. Recommendations and standard operating procedures for intensive care unit and hospital preparations for an influenza epidemic or mass disaster. Intensive Care Med 2010, 36(Suppl 1):S55-S64.

4. Devereaux AV, Dichter JR, Christian MD, Dubler NN, Sandrock CE, Hick JL, Powell T, Geiling JA, Amundson DE, Baudendistel TE, Braner DA, Klein MA, Berkowitz KA, Curtis JR, Rubinson L; Task Force for Mass Critical Care: 
Definitive care for the critically ill during a disaster: a framework for allocation of scarce resources in mass critical care: from a Task Force for Mass Critical Care summit meeting, January 26-27, 2007, Chicago, IL. Chest 2008, 133(5 Suppl):51S-665.

5. Powell T, Christ KC, Birkhead GS: Allocation of ventilators in a public health disaster. Disaster Med Public Health Prep 2008, 2:20-26.

6. Sprung CL, Baras M, lapichino G, Kesecioglu J, Lippert A, Hargreaves C, Pezzi A, Pirracchio R, Edbrooke DL, Pesenti A, Bakker J, Gurman G, Cohen SL, Wiis J, Payen D, Artigas A: The Eldicus prospective, observational study of triage decision making in European intensive care units. Crit Care Med 2012, 40:125-131.

7. White $D B$, Katz MH, Luce JM, Lo B: Who should receive life support during a public health emergency? Using ethical principles to improve allocation decisions. Ann Intern Med 2009, 150:132-138.

8. Christian MD, Hamielec C, Lazar NM, Wax RS, Griffith L, Herridge MS, Lee D, Cook DJ: A retrospective cohort pilot study to evaluate a triage tool for use in a pandemic. Crit Care 2009, 13:R170.

9. Bailey A, Leditschke I, Ranse J, Grove K: Impact of a pandemic triage tool on intensive care admission. Crit Care 2008, 12(Suppl 2):P349.

10. Guest T, Tantam G, Donlin N, Tantam K, Mcmillan H, Tillyard A: An observational cohort study of triage for critical care provision during pandemic influenza: 'clipboard physicians' or 'evidenced based medicine'? Anaesthesia 2009, 64:1199-1206.
11. Shahpori R, Stelfox HT, Doig CJ, Boiteau PJE, Zygun DA: Sequential Organ Failure Assessment in H1N1 pandemic planning. Crit Care Med 2011, 39:827-832.

12. Collaboration TIGHN: InFACT: a global critical care research response to H1N1. Lancet 2010, 375:11-13.

13. Joynt GM, Gomersall CD, Tan P, Lee A, Cheng CA, Wong EL: Prospective evaluation of patients refused admission to an intensive care unit: triage, futility and outcome. Intensive Care Med 2001, 27:1459-1465.

14. Gomersall CD, Joynt GM: What is the benefit in triage? Crit Care Med 2011 39:911-912.

15. Simchen E, Sprung CL, Galai N, Zitser-Gurevich Y, Bar-Lavi Y, Gurman G, Klein M, Lev A, Levi L, Zveibil F, Mandel M, Mnatzaganian G: Survival of critically ill patients hospitalized in and out of intensive care units under paucity of intensive care unit beds. Crit Care Med 2004, 32:1654-1661.

doi:10.1186/cc11842

Cite this article as: Christian MD, et al.: Critical care resource allocation: trying to PREEDICCT outcomes without a crystal ball. Critical Care 2013, 17:107. 